\title{
Development of a Clinical Prediction Model for Infective Endocarditis Among Patients with Undiagnosed Fever: A Pilot Case-Control Study
}

\section{Shun Yamashita (D) \\ Masaki Tago (D) \\ So Motomura \\ Satsuki Oie \\ Hidetoshi Aihara \\ Naoko E Katsuki (D) \\ Shu-ichi Yamashita}

Department of General Medicine, Saga University Hospital, Saga, Japan
Correspondence: Shun Yamashita Department of General Medicine, Saga University Hospital, 5-I-I Nabeshima,

Saga, 849-850 I, Japan

Tel +81952343238

Fax +81952342029

Email sy.hospitalist.japan@gmail.com
Purpose: Infective endocarditis (IE) may be diagnosed as fever of unknown origin due to its delusively non-descriptive clinical features, especially in outpatient clinics. Our objective is to develop a prediction model to discriminate patients to be diagnosed as "definite" IE from "non-definite" by modified Duke criteria among patients with undiagnosed fever, using only history and results of physical examinations and common laboratory examinations.

Patients and Methods: The study was a single-center case-control study. Inpatients at Saga University Hospital diagnosed with IE from 2007 to 2017 and patients with undiagnosed fever from 2015 to 2017 were enrolled. Patients diagnosed with definite IE according to the modified Duke criteria, except those definitely diagnosed with other disorders responsible for fever, were allocated to the IE group. Patients without IE among those defined as non-definite according to the modified Duke criteria were allocated to the undiagnosed fever group. We developed a prediction model to pick up patients who would be "definite" by modified Duke criteria, which was subsequently assessed by area under the curve (AUC).

Results: A total of 144 adult patients were included. Of these, 59 patients comprised the IE group. We developed the prediction model using five indicators, including transfer by ambulance, cardiac murmur, pleural effusion, neutrophil count, and platelet count, with a sensitivity $84.7 \%$, a specificity $84.7 \%$, an AUC 0.893 (95\% confidence interval 0.828 0.959 ), a shrinkage coefficient 0.635 , and a stratum-specific likelihood ratio $0.2-50.4$.

Conclusion: Our prediction model, which uses only indicators easy to gain, facilitates prediction of patients with IE. These indicators can be acquired even at common hospitals and clinics, without requiring advanced medical equipment or invasive examinations.

Trial Registration Number: UMIN000041344.

Keywords: infective endocarditis, undiagnosed fever, case-control study, prediction model, area under the curve

\section{Introduction}

Infective endocarditis (IE) is a diagnostic challenge due to its non-characteristic clinical manifestations and physical and laboratory findings. ${ }^{1}$ IE may be inadequately diagnosed as fever of unknown origin, ${ }^{2,3}$ which is partially due to difficulty in applying all the advanced medical modalities required to make a diagnosis according to the modified Duke criteria (m-DC), such as transesophageal echocardiography (TEE), computed tomography (CT), and magnetic resonance imaging (MRI) for all patients with undiagnosed fever (UF). ${ }^{4}$ This may lead to improper practice of prescribing antibiotics before performing necessary examinations 
including blood cultures, especially at hospitals or clinics without those modalities. Naturally, administration of antibiotics before obtaining blood cultures could lead to failure to detect causative bacteria, causing a delayed diagnosis with poor prognosis of IE. ${ }^{5,6}$ Therefore, predicting patients with a high probability of IE, using data routinely obtained at common outpatient clinics or on admission at most hospitals, could enable timely application of advanced medical examinations and referral to higher level medical institutions, only to appropriate patients.

Although some prediction models have been reported for IE, each of these models has only been applied to one individual bacterium, such as Staphylococcus aureus, ${ }^{7-9}$ Viridans streptococci, ${ }^{10}$ or Enterococcus spp. ${ }^{11}$ Because these models are obviously limited to a single bacterial species, they can only be used after identification of causative bacteria by blood culture. To the best of our knowledge, no prediction models for IE exist, except the Duke criteria and the m-DC, for patients with UF. ${ }^{12}$

We herein report the development of a prediction model for IE among patients with UF, using only simple indicators which can be obtained without advanced medical equipment.

\section{Materials and Methods Study Design and Participants}

The present study was a single-center case-control study. Among patients aged $\geq 20$ years at Saga University Hospital from September 2007 to August 2017, patients with International Statistical Classification of Diseases and Related Health Problems-10 (ICD-10) code I-330, which indicates a diagnosis of IE, were identified. Among patients with I-330, those who did not show a fever $\geq 37{ }^{\circ} \mathrm{C}$ before admission, who were referred for valve surgery after medical treatment for IE at the previous hospital, who had nosocomial onset, and who were not admitted to hospital were excluded. Of the remaining patients, those who were diagnosed as "definite" by $\mathrm{m}-\mathrm{DC}$ as gold standard of diagnosis of IE were allocated to the IE group.

Subsequently, among patients aged $\geq 20$ years at Saga University Hospital from January 2015 to December 2017, those with ICD-10 code R-50-9, which indicates UF, were identified. Among patients with R-50-9, patients without a fever $\geq 37{ }^{\circ} \mathrm{C}$ before admission, patients whose cause of fever was identified at the outpatient department, and patients who were not admitted to hospital were excluded. Of the remaining patients, those with "non-definite" by $\mathrm{m}$-DC were allocated to the UF group. Although patients diagnosed as definite by m-DC among patients with ICD-10 code R-50-9 were principally allocated to the IE group, patients with other definitive diagnoses separate from IE were excluded. The final diagnoses of patients in the UF group were determined according to the ICD-10 based on the diagnoses made and documented in medical records by attending doctors.

The present study was registered at https://www.umin. ac.jp. The design was assessed using the Transparent Reporting of a multivariable prediction model for Individual Prognosis or Diagnosis, also known as TRIPOD. ${ }^{13}$

\section{Setting}

Saga University Hospital is the only university hospital in Saga Prefecture, Kyushu, Japan, which has fully equipped outpatient clinics and 604 inpatient beds across 29 clinical departments, including the departments of general medicine, cardiology, and cardiovascular surgery. The hospital provides high-level medical services mainly for patients in acute disease phases. The Division of Infectious Disease/ Prevention and Control in our hospital usually supervises the diagnosis of infectious diseases, including IE, and chooses appropriate antibiotics and treatment durations for all patients with positive blood culture results, as well as when other departments request consultations.

\section{Data Sources}

Four physicians from the department of general medicine (GP) at our hospital reviewed medical records and referral letters from previous doctors to collect data of potentially eligible patients. Survey items are shown in Supplementary Table 1. Prolonged antimicrobial use for chronic diseases was excluded from the definition of "antibiotic administration before admission." The diagnosis of disordered consciousness was made when patients had a Glasgow Coma Scale score of $\leq 14$ or when people who were around patients on a daily basis felt that something was wrong about the behavior of patients. Presence of pleural effusion or pulmonary edema on chest X-ray or chest $\mathrm{CT}$ on admission was determined by a radiology specialist. If an interpretation by a radiologist was not available, it was determined by two GPs. When the interpretations of the two GPs were in agreement, pleural effusion or pulmonary edema were determined as 
"present." When the interpretations were not in agreement, a final conclusion was reached by discussion between the two GPs. Disseminated infections included pyogenic spondylitis, pyogenic arthritis, spinal epidural abscess, deepseated abscess, and mycotic aneurysm. Disseminated intravascular coagulation (DIC) was diagnosed according to the DIC scoring system of the Japanese Association for Acute Medicine. ${ }^{14}$

\section{Data Analysis}

Missing values were handled as "no abnormalities" for categorical variables and were excluded from the analysis for continuous variables. Categorical variables are expressed as percentages and were compared using the $\chi^{2}$ test. Continuous variables are expressed as median and interquartile range, and were compared using the Mann-Whitney $U$-test. Statistical significance was set at $p<0.05$. In determining covariates of multivariate logistic regression analysis, we excluded items which could not be determined on admission, such as mortality and duration of the hospital stay. We also excluded every item in the m-DC, which we used as the gold standard of the diagnosis of IE, and items with available data less than 50. In light of collinearity, candidate variables that showed significance with a univariate analysis and a low correlation coefficient (in case of a Spearman's $r$ value $>0.7$ or a Kendall's r value $>0.7$ between two items, one factor was selected, and the others were removed) were selected. A backward selection method was used for multivariate logistic regression analysis.

Using the logistic regression model, the predictive probability of definite IE according to the m-DC were calculated. The cut-off values were set to the maximum sum of sensitivity and specificity. The predictive performance of the model was assessed using the area under the curve (AUC) derived from the same cohort. Model calibration was assessed using the shrinkage coefficient ${ }^{15}$ and the HosmerLemeshow test. SPSS Statistics (version 25; IBM Corp., Armonk, NY, USA) was used for statistical analysis.

\section{Ethical Consideration}

The study conformed to Ethical Guidelines for Medical and Health Research Involving Human Subjects in Japan. The ethics committee of Saga University Hospital approved the study (file number: 2020-05-R-04), which was conducted in accordance with the guidelines of the 1975 Declaration of Helsinki. All subjects, who were informed about the purpose of the study via the homepage of clinical research center of Saga University Hospital, provided consent as per the comprehensive agreement method, and patient anonymity was protected.

\section{Results}

\section{Enrollment and Allocation of Study Participants}

Enrollment of participants to the present study and allocation to either the IE group or the UF group are shown in Figure 1. Thirty-three of 108 patients with code I-330, who were without a fever $\geq 37{ }^{\circ} \mathrm{C}(\mathrm{n}=10)$, with referral for valve surgery after medical treatment for IE at the previous hospital $(n=7)$, with nosocomial onset $(n=14)$, and who were not admitted to hospital $(n=2)$, were excluded. Of the remaining 75 patients, 54 patients were diagnosed with definite IE according to the $\mathrm{m}-\mathrm{DC}$ and were allocated to the IE group. Thirty-one of 122 patients with code R-50-9, who were without a fever $\geq 37{ }^{\circ} \mathrm{C}$ before admission $(\mathrm{n}=4)$, whose cause of fever was identified at the outpatient department $(n=27)$, and who were not admitted to hospital $(n=0)$, were excluded. Of the remaining 91 patients, six patients were diagnosed with definite IE according to the m-DC, five of whom were allocated to the IE group. One patient was excluded due to a final diagnosis of adult-onset Still's disease. As a result, there were 59 patients in the IE group and 85 patients in the UF group.

\section{Patient Characteristics}

Patients' characteristics are shown in Table 1. The following survey items were more frequently observed in the IE group: dyspnea (IE: $20 \%$ vs UF: $6 \%, p=0.012$ ); neurological symptoms (34\% vs $9 \%, p<0.001)$, including disordered consciousness, paralysis, or weakness within 2 months before admission; long duration of hospital stay (41 days vs 19 days, $p<0.001)$; transfer by ambulance $(51 \%$ vs $1 \%$, $p<0.001)$; periodontitis ( $30 \%$ vs $5 \%, p=0.027$ ); previous IE ( $5 \%$ vs $0 \%, p=0.041)$; prosthetic valve $(19 \%$ vs $0 \%$, $p<0.001)$; 30 -day mortality $(17 \%$ vs $5 \%, p=0.030)$; inhospital mortality $(17 \%$ vs $6 \%, p=0.044)$. The UF group had a higher percentage of patients who were administered antibiotics before admission ( $56 \%$ vs $77 \%, p=0.030)$ and patients with joint or back pain within 2 months before admission ( $17 \%$ vs $35 \%, p=0.008)$. As for vital signs, the IE group had a higher pulse rate (102 bpm vs $90 \mathrm{bpm}$, $p=0.004)$ and respiratory rate (23 breaths/min vs 18 breaths/min, $p<0.001$ ), with higher systemic inflammatory response syndrome ${ }^{16}$ ( 2 vs $\left.1, p<0.001\right)$ and quick sequential organ failure assessment $^{17}$ (1 vs $\left.0, p<0.001\right)$ scores. As for 


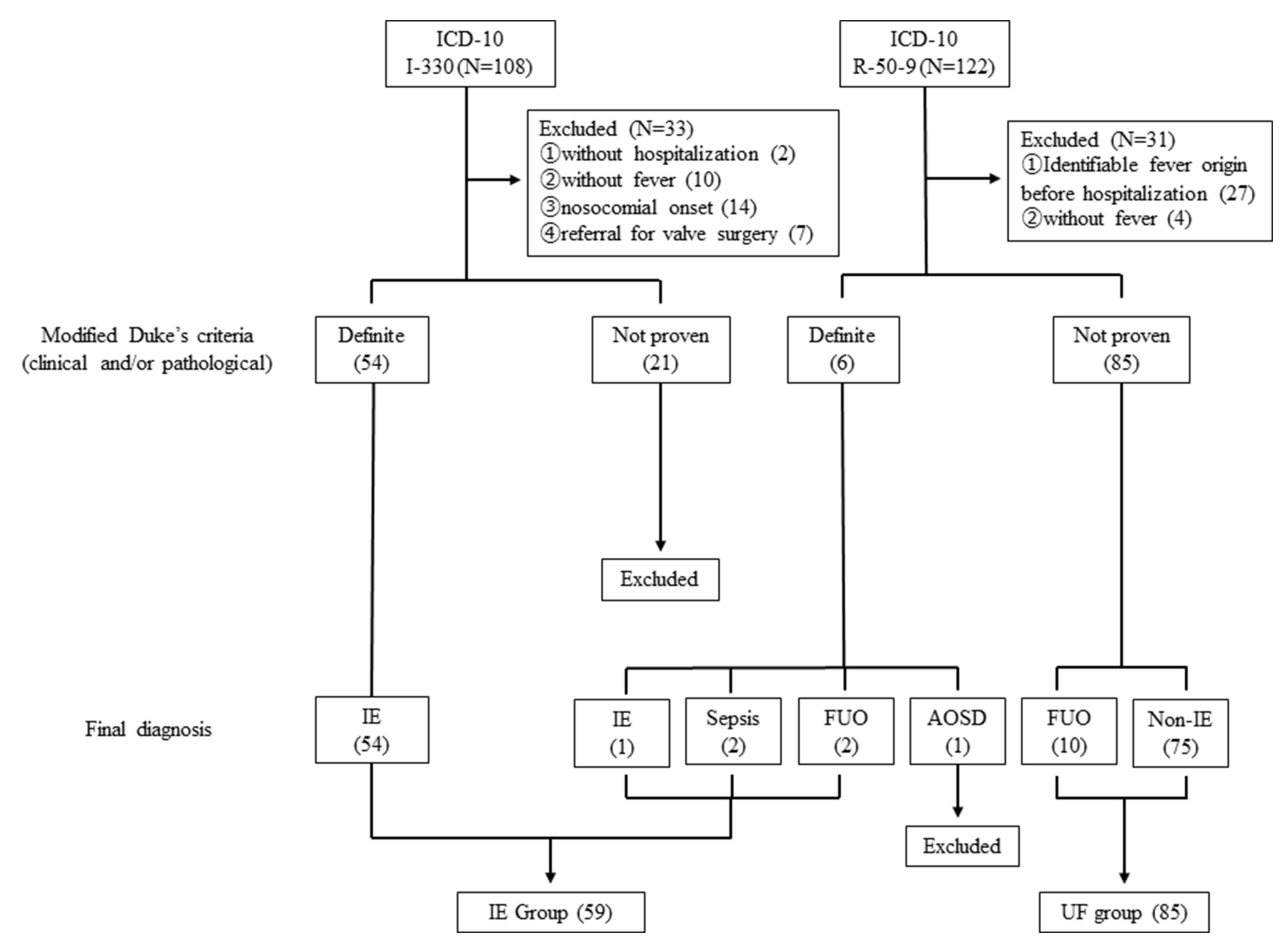

Figure I Enrollment and allocation flow diagram. Thirty-three of 108 patients had International Statistical Classification of Diseases and Related Health Problems-I0 (ICD10) code I-330. Among them, patients without a fever $\geq 37{ }^{\circ} \mathrm{C}(n=10)$, referred for valve surgery after medical treatment for infective endocarditis (IE) at the previous hospital $(n=7)$, with nosocomial onset $(n=14)$, and who were not admitted to hospital $(n=2)$ were excluded. Of the remaining 75 patients, 54 patients were diagnosed with definite IE according to the modified Duke criteria and were allocated to the IE group. Thirty-one of I 22 patients had ICD-I0 code R-50-9. Among them, patients without a fever $\geq 37{ }^{\circ} \mathrm{C}$ before admission $(n=4)$, whose cause of fever was identified at the outpatient department ( $\left.n=27\right)$, and who were not admitted to hospital ( $n=0$ ) were excluded. Of the remaining 91 patients, six patients were diagnosed with definite IE according to the modified Duke criteria. Five patients were allocated to the IE group, and one patient was excluded with a final diagnosis of adult-onset Still's disease. As a result, 59 patients and 85 patients were enrolled in the IE group and the undiagnosed fever group, respectively.

physical examinations, the IE group had a greater percentage of patients with cardiac murmur ( $53 \%$ vs $9 \%, p<0.001)$. No difference was observed in the percentage of patients with limb edema ( $22 \%$ vs $15 \%, p=0.301)$. The execution rate of each investigation, breakdown of final diagnoses in the UF group, and causative bacteria in the IE group are shown in Supplementary Tables $2-4$, respectively.

\section{Laboratory Findings on Admission to Our Hospital}

Laboratory findings on admission are shown in Table 2 . White blood cell count (IE: $13.5 \times 10^{3} / \mu \mathrm{L}$ vs UF: $10.5 \times 10^{3}$ / $\mu \mathrm{L}, \quad p=0.004)$, neutrophil count $(89.6 \%$ vs $80.4 \%$, $p<0.001)$, D-dimer concentration $(8.2 \mu \mathrm{g} / \mathrm{mL}$ vs $3.3 \mu \mathrm{g} /$ $\mathrm{mL}, p=0.003)$, total bilirubin concentration $(0.9 \mathrm{mg} / \mathrm{dL}$ vs $0.6 \mathrm{mg} / \mathrm{dL}, p=0.001)$, lactate dehydrogenase concentration (337 IU/L vs $220 \mathrm{IU} / \mathrm{L}, p<0.001$ ), blood urea nitrogen concentration $(24.1 \mathrm{mg} / \mathrm{dL}$ vs $16.0 \mathrm{mg} / \mathrm{dL}, p<0.001)$, and serum creatinine concentration $(1.1 \mathrm{mg} / \mathrm{dL}$ vs $0.8 \mathrm{mg} / \mathrm{dL}$, $p=0.012$ ) were higher in the IE group compared with the
UF group. Platelet count $\left(11.7 \times 10^{4} / \mu \mathrm{L}\right.$ vs $27.9 \times 10^{4} / \mu \mathrm{L}$, $p<0.001)$ and serum albumin concentration $(2.5 \mathrm{~g} / \mathrm{dL}$ vs $2.8 \mathrm{~g} / \mathrm{dL}, p=0.033$ ) were lower in the IE group compared with the UF group. Additionally, the frequency of DIC ( $37 \%$ vs $12 \%, p=0.001$ ) was higher in the IE group compared with the UF group.

\section{Imaging Studies}

The results of imaging studies are shown in Table 3 . Although there was no difference in the morbidity of total valvular regurgitation, mitral regurgitation (IE: $59 \%$ vs UF: $40 \%, p=0.017$ ) was higher in the IE group, and tricuspid regurgitation (29\% vs $51 \%$, $p=0.029)$ and pulmonary regurgitation ( $7 \%$ vs $26 \%$, $p=0.007)$ were higher in the UF group. No differences were observed in the percentage of patients with an ejection fraction $\leq 50 \%$ within 14 days after admission $(15 \%$ vs $6 \%, p=0.083)$ and metastatic infection $(14 \%$ vs $7 \%, p=0.239)$. Pulmonary edema $(29 \%$ vs $1 \%, p<0.001)$ and pleural effusion $(64 \%$ vs $29 \%, p<0.001)$ on admission were higher in the IE group. Chest X-ray or CT 
Table I Univariate Analysis of Patient Characteristics

\begin{tabular}{|c|c|c|c|}
\hline & IE (N=59) & UF $(\mathbf{N}=85)$ & $p$ value \\
\hline Age, years & 71 (56-77) & $66(51-78)$ & 0.461 \\
\hline Aged $>60$ years & $34(58)$ & $41(48)$ & 0.438 \\
\hline Male & $30(5 \mathrm{I})$ & $42(49)$ & 0.847 \\
\hline Duration of hospital stay & $41(30-59)$ & $19(11-29)$ & $<0.001$ \\
\hline \multicolumn{4}{|l|}{ Mortality } \\
\hline 30-day mortality & $9 / 53(17)$ & $4(5)$ & 0.030 \\
\hline In-hospital mortality & $10(17)$ & $5(6)$ & 0.044 \\
\hline Transfer by ambulance & $30(5 \mathrm{I})$ & $I(I)$ & $<0.001$ \\
\hline Administration of antibiotics before admission & $33(56)$ & $56 / 73(77)$ & 0.030 \\
\hline \multicolumn{4}{|l|}{ Symptoms within 2 months } \\
\hline Malaise & $17(29)$ & $33(39)$ & 0.126 \\
\hline Dyspnea & $12(20)$ & $5(6)$ & 0.012 \\
\hline Joint/back pain & $10(17)$ & $30(35)$ & 0.008 \\
\hline Disordered consciousness & $15(25)$ & $6(7)$ & 0.003 \\
\hline Paralysis/weakness & $9(15)$ & $2(2)$ & 0.006 \\
\hline Neurological symptoms ${ }^{\dagger}$ & $20(34)$ & $8(9)$ & $<0.001$ \\
\hline \multicolumn{4}{|l|}{ Past medical history } \\
\hline Previous IE & $3(5)$ & NA & 0.041 \\
\hline Prosthetic valve & $11(19)$ & NA & $<0.001$ \\
\hline Chronic dermatological disorder & $7(12)$ & $7(8)$ & 0.544 \\
\hline Diabetes mellitus & $12(20)$ & $15(18)$ & 0.812 \\
\hline Use of steroids & $5(9)$ & $8(9)$ & 0.993 \\
\hline Use of immunosuppressant agents & $3(5)$ & $3(4)$ & 0.701 \\
\hline Malignancy & $4(7)$ & II (I3) & 0.190 \\
\hline Dental problems ${ }^{\dagger \dagger}$ & $22 / 23(96)$ & $9 / 21(43)$ & $<0.001$ \\
\hline Periodontitis & $7 / 23(30)$ & $|/ 2|(5)$ & 0.027 \\
\hline Dentistry going to hospital within 6 months & $10 / 32(31)$ & $5 / 9(56)$ & 0.181 \\
\hline Invasive dental treatment history within 6 months & $9 / 54(17)$ & $3 / 7(43)$ & 0.101 \\
\hline
\end{tabular}

Notes: Categorical data are expressed as $\mathrm{n}(\%)$ and were compared using the $\chi^{2}$ test. Continuous variables are expressed as median (interquartile range) and were compared using the Mann-Whitney U-test. 'Including disordered consciousness, paralysis, and weakness. ${ }^{\dagger \dagger}$ Including caries, periodontitis, tooth defect, and poor oral hygiene.

Abbreviations: SIRS, systemic inflammatory response syndrome; qSOFA, quick Sequential [Sepsis-related] Organ Failure Assessment.

findings of 87 patients $(60.4 \%)$, specifically 37 of 59 patients $(62.7 \%)$ in the IE group and 50 of 85 patients $(58.8 \%)$ in the UF group, were interpreted by a radiologist. Five patients underwent neither chest $\mathrm{X}$-ray nor CT. Two GPs interpreted the chest X-rays of the remaining 52 patients with concordance rates for pleural effusion and pulmonary edema of $90.4 \%$ and $84.6 \%$, respectively.

\section{Multivariate Logistic Regression Model}

The results of the multivariate logistic regression analysis are shown in Table 4. Transfer by ambulance (odds ratio
[OR] 59.1, 95\% confidence interval [CI] 3.78-922.52), cardiac murmur (OR 17.2, 95\% CI 4.49-65.81), pleural effusion (OR 4.2, 95\% CI 1.33-13.39), neutrophil count (OR 1.1, 95\% CI 1.03-1.16), and platelet count (OR 0.9, 95\% CI 0.91-0.98) were significant factors for the prediction of IE.

\section{Performance of the Predictive Model}

The detailed prediction model is shown in online Supplementary Appendix 1. The AUC was 0.893 (95\% CI 0.828-0.959: Figure 2). The model was well calibrated using the Hosmer-Lemeshow test $(p=0.440)$ with 
Table 2 Univariate Analysis of Laboratory Findings

\begin{tabular}{|c|c|c|c|}
\hline & IE (N=59) & UF $(N=85)$ & $p$ value \\
\hline White blood cell count $\left(\times 10^{3} / \mu \mathrm{L}\right)$ & | $3.5(9.3-\mid 7.9)$ & $10.5(7.6-13.2)$ & 0.004 \\
\hline Neutrophil count (\%) & $89.6(79.4-93.0)$ & $80.4(71.9-85.7)$ & $<0.001$ \\
\hline Platelet count $\left(\times 10^{4} / \mu \mathrm{L}\right)$ & $11.7(8.0-21.8)$ & $27.9(|7|-4 \mid .9)$. & $<0.001$ \\
\hline $\mathrm{FDP}(\mu \mathrm{g} / \mathrm{mL})$ & $19.6(10.0-42.1)$ & $9.7(6.1-29.1)$ & 0.060 \\
\hline D-dimer $(\mu \mathrm{g} / \mathrm{mL})$ & $8.2(3.5-20.1)$ & $3.3(1.5-9.3)$ & 0.003 \\
\hline PT-INR & $1.2(1.1-1.4)$ & $1.2(1.1-1.2)$ & 0.014 \\
\hline Albumin $(g / d L)$ & $2.5(2.1-3.0)$ & $2.8(2.3-3.3)$ & 0.033 \\
\hline Total bilirubin $(\mathrm{mg} / \mathrm{dL})$ & $0.9(0.6-1.3)$ & $0.6(0.4-0.9)$ & 0.001 \\
\hline AST (IU/L) & $38.0(25.0-68.5)$ & $30.0(18.3-53.5)$ & 0.090 \\
\hline ALT (IU/L) & $22.5(15.0-49.2)$ & $26.5(14.0-50.3)$ & 0.619 \\
\hline LDH (IU/L) & $337.0(262.3-434.0)$ & $220.0(165.0-330.8)$ & $<0.001$ \\
\hline BUN (mg/dL) & $24.1(14.8-40.1)$ & $16.0(10.8-22.7)$ & $<0.001$ \\
\hline Creatinine $(\mathrm{mg} / \mathrm{dL})$ & I.I (0.7-I.8) & $0.8(0.6-1.0)$ & 0.012 \\
\hline Sodium (mEq/L) & $136.0(|3| .3-139.0)$ & $136.0(133.0-139.0)$ & 0.267 \\
\hline Potassium (mEq/L) & $4.1(3.4-4.6)$ & $4.0(3.7-4.4)$ & 0.868 \\
\hline Chloride (mEq/L) & $99.0(96.0-104.0)$ & $99.0(96.3-101.0)$ & 1.000 \\
\hline C-reactive protein $(\mathrm{mg} / \mathrm{dL})$ & $10.4(4.6-17.2)$ & $11.7(3.9-17.6)$ & 0.685 \\
\hline $\mathrm{DIC}^{\dagger}$ & $22(37)$ & $10(12)$ & 0.001 \\
\hline
\end{tabular}

Notes: Categorical data are expressed as $\mathrm{n}(\%)$ and were compared using the $\chi^{2}$ test. Continuous variables are expressed as median (interquartile range) and were compared using the Mann-Whitney U-test. ${ }^{\dagger} n$ (\%), patients who fulfilled the DIC scoring system of the Japanese Association for Acute Medicine.

Abbreviations: ALT, alanine aminotransferase; AST, aspartate aminotransferase; BUN, blood urea nitrogen; FDP, fibrin/fibrinogen degradation products; LDH, lactate dehydrogenase; PT-INR, prothrombin time-international normalized ratio; DIC, disseminated intravascular coagulation.

Table 3 Univariate Analysis of Imaging Studies

\begin{tabular}{|c|c|c|c|}
\hline & IE $(\mathrm{N}=59)$ & UF $(N=85)$ & $p$ value \\
\hline \multirow{2}{*}{\multicolumn{4}{|c|}{$\begin{array}{l}\text { Echocardiographic } \\
\text { findings }\end{array}$}} \\
\hline & & & \\
\hline Ejection fraction $<50 \%^{\dagger}$ & $9(15)$ & $3 / 53(6)$ & 0.083 \\
\hline Valvular regurgitation & $45(76)$ & $37 / 53(70)$ & 0.145 \\
\hline Aortic regurgitation & $18(31)$ & $1 \mathrm{I} / 53(2 \mathrm{I})$ & 0.179 \\
\hline Mitral regurgitation & $35(59)$ & $2 \mathrm{I} / 53(40)$ & 0.017 \\
\hline Tricuspid regurgitation & $17(29)$ & $27 / 53(5 I)$ & 0.029 \\
\hline Pulmonary regurgitation & $4(7)$ & $14 / 53(26)$ & 0.007 \\
\hline \multicolumn{4}{|l|}{$\begin{array}{l}\text { Radiological imaging } \\
\text { findings }\end{array}$} \\
\hline Pulmonary edema & $17(29)$ & $1 / 72(1)$ & $<0.001$ \\
\hline Pleural effusion & $38(64)$ & $23 / 72(29)$ & $<0.001$ \\
\hline Metastatic infection & $8(14)$ & $6(7)$ & 0.239 \\
\hline Deep-seated abscess & $7 / 39$ (I8) & $3 / 52(6)$ & 0.066 \\
\hline Pyogenic arthritis & $3 / 40(8)$ & $|/ 5|(2)$ & 0.201 \\
\hline Vertebral osteomyelitis & $4 / 8(50)$ & $4 / 12(33)$ & 0.456 \\
\hline Spinal epidural abscess & $1 / 8(13)$ & $1 / / 2(8)$ & 0.761 \\
\hline
\end{tabular}

Notes: Categorical data are expressed as $\mathrm{n}(\%)$ and were compared using the $\chi^{2}$ test. 'Within 2 weeks after administration.

a shrinkage coefficient of 0.635 (Figure 3). The stratumspecific likelihood ratio was $0.2-50.4$, increasing as the score increased (Table 5). The cut-off value of the prediction model was -1.1 , with a sensitivity of $84.7 \%$ and a specificity of $84.7 \%$.
Table 4 The Results of the Multivariate Logistic Regression Analysis

\begin{tabular}{|l|c|c|c|}
\hline Predictor Variables & OR & $95 \% \mathbf{C I}$ & $\boldsymbol{p}$ value \\
\hline Transfer by ambulance & 59.1 & $3.78-922.52$ & 0.004 \\
Cardiac murmur on admission & 17.2 & $4.49-65.81$ & $<0.00 \mathrm{I}$ \\
Pleural fluid & 4.2 & $1.33-13.39$ & 0.014 \\
Neutrophil count $(\%)$ & 1.1 & $1.03-1.16$ & 0.002 \\
Platelet count $\left(\times 10^{4} / \mu \mathrm{L}\right)$ & 0.9 & $0.91-0.98$ & 0.004 \\
\hline
\end{tabular}

Abbreviations: OR, odds ratio; $\mathrm{Cl}$, confidence interval.

\section{Discussion}

IE can be misdiagnosed as fever of unknown origin, especially at average hospitals or outpatient clinics that are unequipped with advanced medical modalities, such as MRI, CT, or TEE. Although the m-DC requires such investigations to be performed, ${ }^{4}$ it could be unrealistic to apply expensive and possibly invasive medical modalities to all patients with UF. Therefore, it is of profound significance to select patients with a high probability of IE using indicators that are easy to obtain in common practice, making it possible to choose appropriate patients to undergo such examinations or to be referred to advanced medical institutions. We developed a prediction model for IE among patients with UF using five simple indicators, transfer by ambulance, cardiac murmur, pleural effusion, 


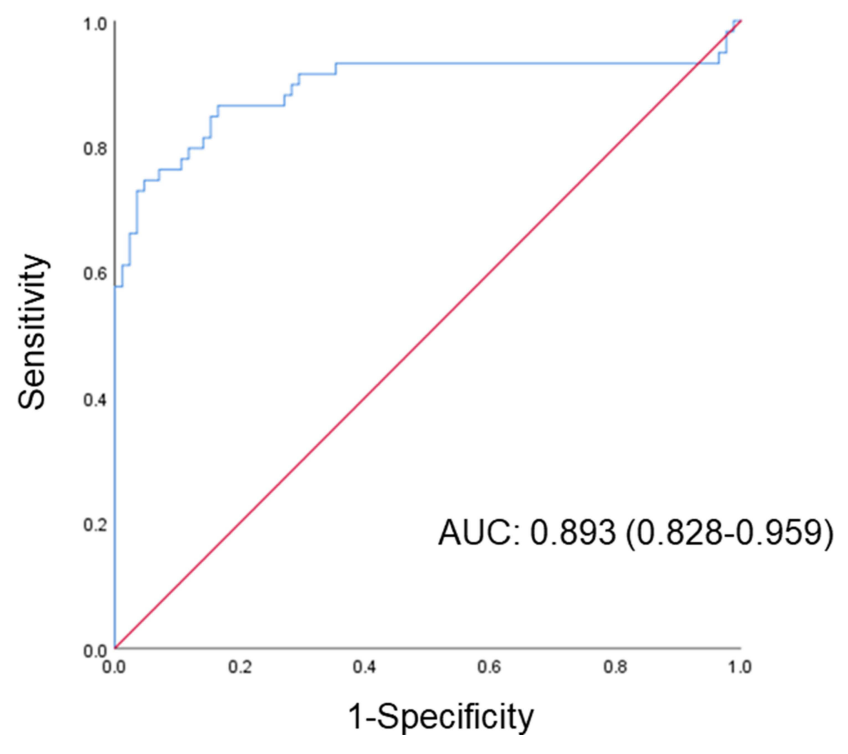

Figure 2 Receiver operating characteristic curve and AUC. The predictive performance of the model was assessed by the area under the curve (AUC) derived from the same cohort. The AUC was 0.893 (95\% confidence interval 0.828-0.959).

neutrophil count, and platelet count. These indicators can be obtained at common hospitals and clinics, without requiring advanced medical equipment or invasive examinations. Furthermore, our model could identify or exclude definite IE diagnosed according to the m-DC.
Table 5 Stratified Likelihood Ratio of Our Clinical Prediction Model

\begin{tabular}{|l|c|c|c|}
\hline Score & Likelihood Ratio & IE & UF \\
\hline-8.5 to -3.2 & 0.2 & 4 & 32 \\
-3.2 to -1.5 & 0.2 & 4 & 32 \\
-1.3 to 1.3 & 1.2 & 16 & 20 \\
I.5 to 8.4 & 50.4 & 35 & I \\
\hline
\end{tabular}

Abbreviations: IE, infected endocarditis; UF, undiagnosed fever.

In the present study, transfer by ambulance was one of the predictors of IE. However, it could be inappropriate to depreciate patients visiting without ambulance because this is one of the factors that delays IE diagnosis. ${ }^{18}$ In that respect, our model could identify patients with the risk of IE among those who visit hospital without ambulance, because our model was proved to be sufficiently accurate using the same subjects, in which approximately half of them visited without ambulance. Furthermore, a body temperature $\leq 38{ }^{\circ} \mathrm{C}$ and a serum C-reactive protein (CRP) concentration $<10 \mathrm{mg} / \mathrm{dL}$ also delay IE diagnosis. ${ }^{18}$ Our prediction model can identify patients with such delaying factors because our patients had a fever $\geq 37{ }^{\circ} \mathrm{C}$ before admission, with $44 \%$ of patients in the IE group showing

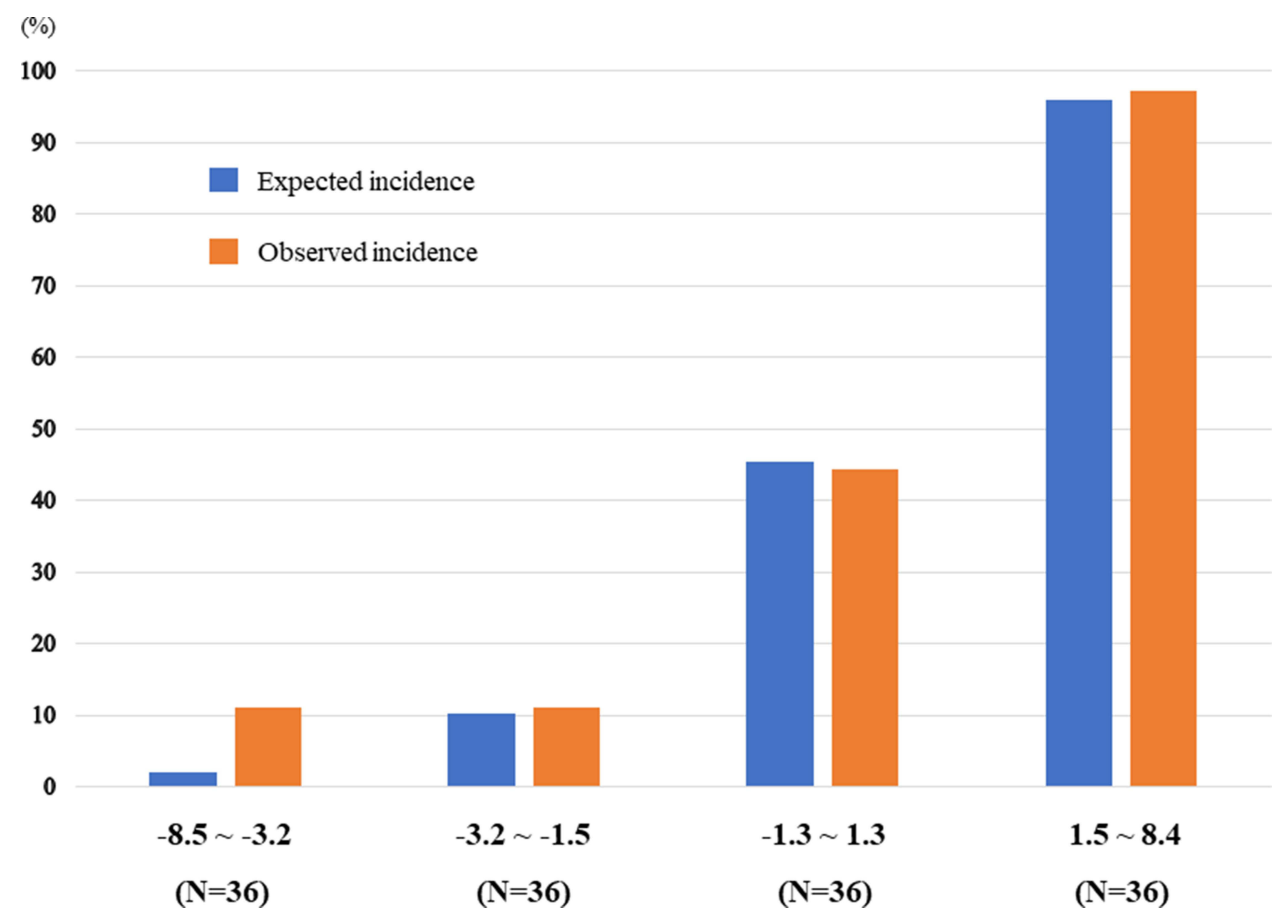

Figure 3 Predictive and observed rates of IE in quadrisect groups according to scores in the internal validation cohort. No difference was found between the expected rate derived from the prediction model and the actual ratio observed in the IE group in each of the four groups with accurate calibration. 
a serum CRP concentration $<10 \mathrm{mg} / \mathrm{dL}$. A similar degree of accuracy was observed using these same subjects.

Even using modernized antibiotics, mortality rates from IE are as high as $11 \%-30 \%{ }^{19-21}$ Furthermore, administration of antibiotics before obtaining blood cultures could cause negative results, which could delay diagnosis. ${ }^{6,18}$ Using our prediction model before administration of antibiotics could reduce inappropriate administration of antibiotics prior to obtaining blood cultures by alerting clinicians of a possible IE diagnosis.

One drawback of our study is that IE on prosthetic valves was diagnosed using the m-DC. When diagnosing IE on prosthetic valves, the sensitivity of the m-DC was as low as $50 \%$, ${ }^{22}$ which could be improved by combined use of ${ }^{18} \mathrm{~F}$-fluorodeoxyglucose positron emission tomography with $\mathrm{CT}$ or single-photon emission $\mathrm{CT}^{23,24}$ However, application of these modalities is unrealistic to diagnose IE in Japan due to Japanese Health Insurance system. Thus, further study is required to determine whether our prediction model is useful for IE on prosthetic valves.

The present study had several limitations. Because the annual incidence of IE was low, ${ }^{25}$ the number of samples that were able to be collected in a single-center pilot case-control study such as the present study was limited. The use of ambulance, which was one of the predictors of IE in the present study, might have differed among regions, countries, or health care services. Therefore, multi-center studies are required to clarify whether the factor of transfer by ambulance can be widely used. Finally, external validation is preferable because the AUC of our prediction model was derived using the same cohort as was used to develop the model.

\section{Conclusion}

We developed a prediction model for infective endocarditis in patients with undiagnosed fever using only easily obtainable data, transfer by ambulance, cardiac murmur, pleural effusion, neutrophil count, and platelet count. Our prediction model could make early detection of patients with IE possible, resulting in proper treatments and reducing inappropriate practices, such as administration of antibiotics prior to obtaining blood cultures.

\section{Abbreviations}

IE, infective endocarditis; m-DC, modified Duke criteria; TEE, transesophageal echocardiography; CT, computed tomography; MRI, magnetic resonance imaging; UF, undiagnosed fever; ICD-10, International Statistical Classification of Diseases and Related Health Problems-
10; GP, physician from the department of general medicine; DIC, disseminated intravascular coagulation; AUC, area under the curve; OR, odds ratio; $\mathrm{CI}$, confidence interval; CRP, C-reactive protein.

\section{Data Sharing Statement}

The datasets of the present study are available from the UMIN database, https://upload.umin.ac.jp/cgi-open-bin /icdr_e/ctr_view.cgi?recptno=R000047202.

\section{Acknowledgments}

We thank Edanz Group for editing a draft of this manuscript.

\section{Funding}

This research received no specific grant from any funding agency in the public, commercial or not-for-profit sectors.

\section{Disclosure}

The authors report no conflicts of interest in this work.

\section{References}

1. Cahill TJ. Bernard DP. Infective endocarditis. Lancet. 2016;387: 882-893. doi:10.1016/S0140-6736(15)00067-7

2. Naito T, Tanei M, Ikeda N, et al. Key diagnostic characteristics of fever of unknown origin in Japanese patients: a prospective multicentre study. BMJ Open. 2019;9(11):e032059. doi:10.1136/bmjopen2019-032059

3. Ikuni Y, Okada J, Kondo H, Kashiwazaki S. Current fever of unknown origin 1982-1992. Intern Med. 1994;33(2):67-73. doi:10. 2169/internalmedicine.33.67

4. Li JS, Sexton DJ, Mick N, et al. Proposed modifications to the Duke criteria for the diagnosis of infective endocarditis. Clin Infect Dis. 2000;30(4):633-638. doi:10.1086/313753

5. Jingushi N, Iwata M, Terasawa T. Clinical features of patients with infective endocarditis presenting to the emergency department: a retrospective case series. Nagoya J Med Sci. 2017;79:467-476.

6. Fukuchi T, Iwata K, Ohji G. Failure of early diagnosis of infective endocarditis in Japan-a retrospective descriptive analysis. Medicine. 2014;93(27):e237. doi:10.1097/MD.0000000000000237

7. Bai AD, Agarwal A, Steinberg M, et al. Clinical predictors and clinical prediction rules to estimate initial patient risk for infective endocarditis in Staphylococcus aureus bacteraemia: a systematic review and meta-analysis. Clin Microbiol Infect. 2017;23(12): 900-906. doi:10.1016/j.cmi.2017.04.025

8. Palraj BR, Baddour LM, Hess EP, Steckelberg JM. Predicting risk of endocarditis using a clinical tool (PREDICT): scoring system to guide use of echocardiography in the management of staphylococcus aureus bacteremia. Clin Infect Dis. 2015;61(1):18-28. doi:10.1093/ $\mathrm{cid} / \mathrm{civ} 235$

9. Gow N, Lowe BS, Freeman J, Roberts S. The role of echocardiography in Staphylococcus aureus bacteraemia at Auckland City Hospital. N Z Med J. 2015;128(1416):28-35.

10. Sunnerhagen T, Törnell A, Vikbrant M, Nilson B, Rasmussen M. HANDOC: a handy score to determine the need for echocardiography in non- $\beta$-hemolytic streptococcal bacteremia. Clin Infect Dis. 2018;66(5):693-698. doi:10.1093/cid/cix880 
11. Bouza E, Kestler M, Beca T, et al. The NOVA score: a proposal to reduce the need for transesophageal echocardiography in patients with enterococcal bacteremia. Clin Infect Dis. 2015;60(4):528-535. doi: $10.1093 / \mathrm{cid} / \mathrm{ciu} 872$

12. Hoen B, Béguinot I, Rabaud C, et al. The Duke criteria for diagnosing infective endocarditis are specific: analysis of 100 patients with acute fever or fever of unknown origin. Clin Infect Dis. 1996;23 (2):298-302. doi:10.1093/clinids/23.2.298

13. Collins GS, Reitsma JB, Altman DG, Moons KG. Transparent reporting of a multivariable prediction model for individual prognosis or diagnosis (TRIPOD): the TRIPOD statement. BMJ. 2015;350:g7594. doi:10.1136/bmj.g7594

14. Gando S, Saitoh D, Ogura H, et al. Natural history of disseminated intravascular coagulation diagnosed based on the newly established diagnostic criteria for critically ill patients: results of a multicenter, prospective survey. Crit Care Med. 2008;36(1):145-150. doi:10. 1097/01.CCM.0000295317.97245.2D

15. Harrell FE Jr, Lee KL, Mark DB. Multivariable prognostic models: issues in developing models, evaluating assumptions and adequacy, and measuring and reducing errors. Stat Med. 1996;15(4):361-387. doi:10.1002/(SICI)1097-0258(19960229)15:4<361::AID-SIM168>3. $0 . \mathrm{CO} ; 2-4$

16. Bone RC, Balk RA, Cerra FB, et al. Definitions for sepsis and organ failure and guidelines for the use of innovative therapies in sepsis The ACCP/SCCM Consensus Conference Committee. American College of Chest Physicians/Society of Critical Care Medicine. Chest. 1992;101(6):1644-1655. doi:10.1378/chest.101.6.1644

17. Seymour CW, Liu VX, Iwashyna TJ, et al. Assessment of clinical criteria for sepsis: for the Third International consensus definitions for sepsis and septic shock (sepsis-3). JAMA. 2016;315(8):762-774. doi:10.1001/jama.2016.0288

18. Nishiguchi S, Nishino K, Kitagawa I, Tokuda Y. Factors associated with delayed diagnosis of infective endocarditis. A retrospective cohort study in a teaching hospital in Japan. Medicine. 2020;99(30): e21418. doi:10.1097/MD.0000000000021418
19. Nakatani S, Mitsutake K, Ohara T, Kokubo Y, Yamamoto H, Hanai S; CADRE Investigators. Recent picture of infective endocarditis in Japan-lessons from Cardiac Disease Registration (CADRE-IE). Circ J. 2013;77:1558-1564. doi:10.1253/circj.CJ-12-1101

20. Mostaghim AS, Lo HYA, Khardori N. A retrospective epidemiologic study to define risk factors, microbiology, and clinical outcomes of infective endocarditis in a large tertiary-care teaching hospital. $S A G E$ Open Med. 2017;5:205031211774177. doi:10.1177/2050312117741 772

21. García-Cabrera E, Fernández-Hidalgo N, Almirante B, et al.; Group for the Study of Cardiovascular Infections of the Andalusian Society of Infectious Diseases. Neurological complications of infective endocarditis: risk factors, outcome, and impact of cardiac surgery: a multicenter observational study. Circulation. 2013;127: 2272-2284. doi:10.1161/CIRCULATIONAHA.112.000813

22. Vincent LL, Otto CM. Infective endocarditis: update on epidemiology, outcomes, and management. Curr Cardiol Rep. 2018;20(10):86. doi:10.1007/s11886-018-1043-2

23. Pizzi MN, Roque A, Fernández-Hidalgo N, et al. Improving the diagnosis of infective endocarditis in prosthetic valves and intracardiac devices with $18 \mathrm{~F}$-fluordeoxyglucose positron emission tomography/ computed tomography angiography initial results at an infective endocarditis referral center. Circulation. 2015;132(12):1113-1126. doi:10.1161/CIRCULATIONAHA.115.015316

24. Habib G, Lancellotti P, Antunes MJ, et al. 2015 ESC guidelines for the management of infective endocarditis: the task force for the management of infective endocarditis of the European Society of Cardiology (ESC) endorsed by: european Association for Cardio-Thoracic Surgery (EACTS), the European Association of Nuclear Medicine (EANM). Eur Heart J. 2015;36(44):3075-3128.

25. Selton-Suty C, Célard M, Le Moing V, et al. Preeminence of Staphylococcus aureus in infective endocarditis: a 1-year population-based survey. Clin Infect Dis. 2012;54(9):1230-1239. doi: $10.1093 / \mathrm{cid} / \mathrm{cis} 199$
International Journal of General Medicine

\section{Publish your work in this journal}

The International Journal of General Medicine is an international, peer-reviewed open-access journal that focuses on general and internal medicine, pathogenesis, epidemiology, diagnosis, monitoring and treatment protocols. The journal is characterized by the rapid reporting of reviews, original research and clinical studies

\section{Dovepress}

across all disease areas. The manuscript management system is completely online and includes a very quick and fair peer-review system, which is all easy to use. Visit http://www.dovepress.com/ testimonials.php to read real quotes from published authors. 